DE LA RECHERCHE A L' INDUSTRIE
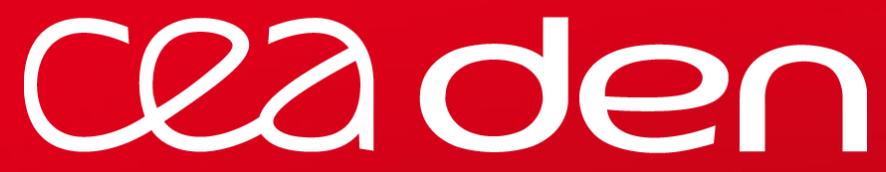

Ecole Nationale 【] Supérieure des Mines บ' SAINT-ETIENNE

\section{ANALYSIS OF SELECTIVE LASER MELTING OF RESORBABLE BIOCERAMICS}
Pascal Aubry ${ }^{1}$, Olivier Hercher ${ }^{1}$, Didier Nimal ${ }^{2}$, David Marchat ${ }^{3}$

${ }_{1}^{1}$ CEA/DEN/DANS/DPC/SEARS/LISL, CEA Saclay, 91191 Gif-surYvette Cedex, France

${ }^{2}$ Osseomatrix, 9100 Evry, France

${ }^{3}$ Ecole Nationale Supérieure des Mines de Saint-Etienne, Centre Ingénierie et Santé, UMR CNRS 6638, Saint-Etienne, France 


\section{OBJECTIVES OF THE PROJECT}

WHAT COULD BE POSSIBLE IN A NEAR FUTURE... 


\section{OBJECTIVES OF THE PROJECT}

\section{DESIGN/MANUFACTURING OF BIOCERAMIC IMPLANT}

- Better Osteointegration of the Implant (Compared to Metallic Implants)

- Implant as a Bone-repair-kit : Induction of Natural Bone Regeneratinon by the Implant

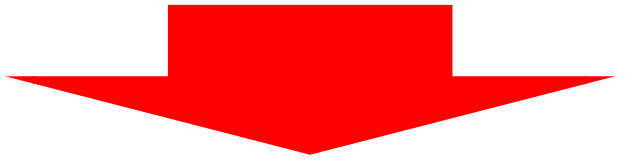

\section{OrthoFlase Project}

\section{Projet ANR-11-TECS-0005:OrthoFlase}

SMES

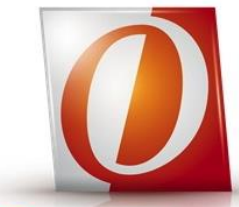

OsseoMatrix

Project leader

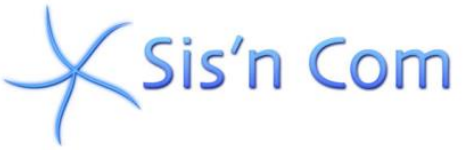

Web-based software and data management

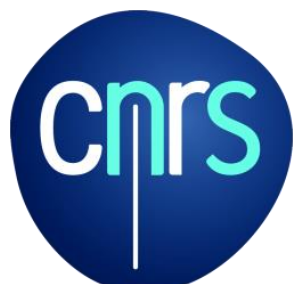

Biocompatibility
Ecole Nationale SAINT-ETIENNE

Powder

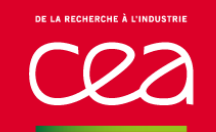

Process development 


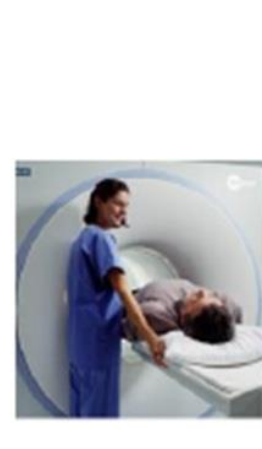

scanning

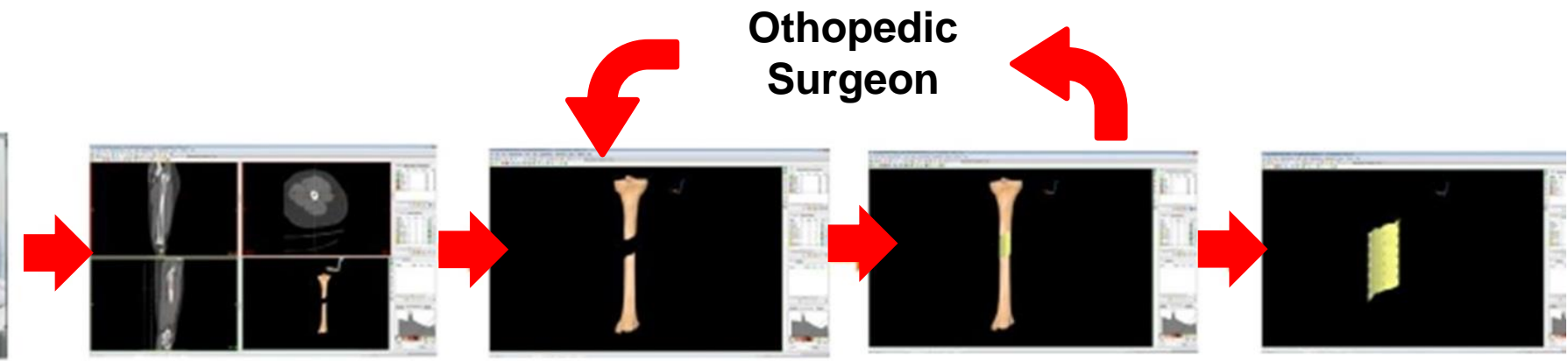

Data processing and CAD generation

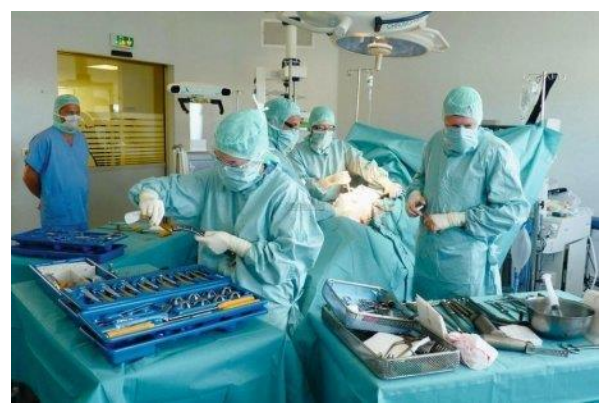

Surgery
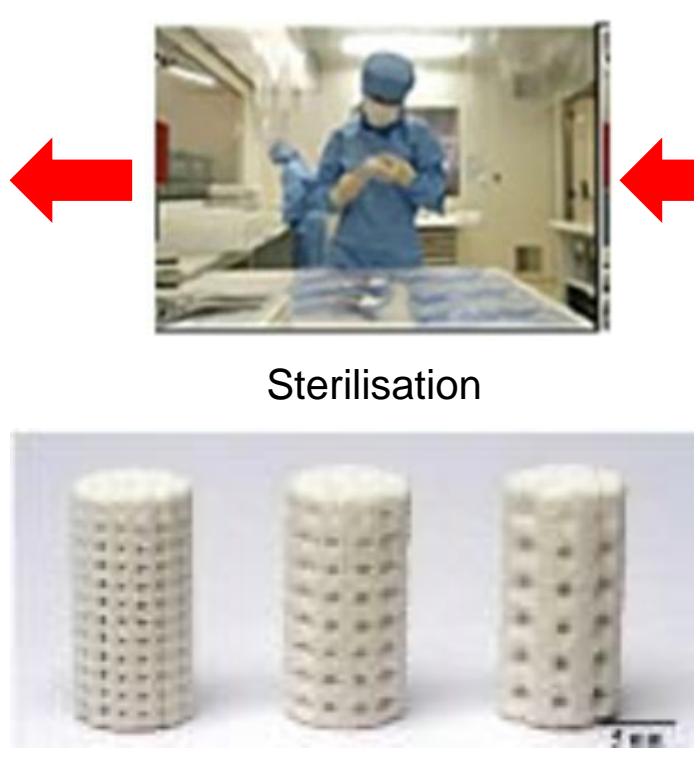

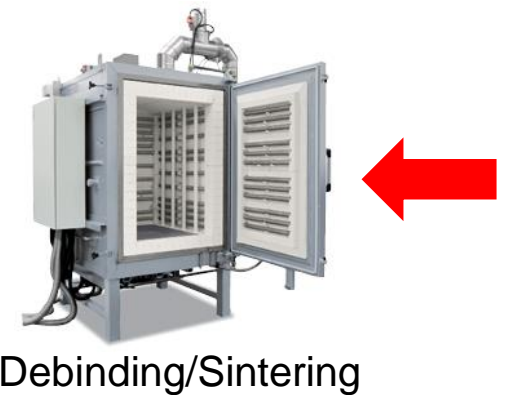

Debinding/Sintering

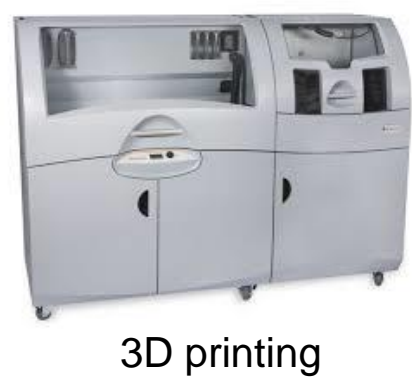

- HAP (Hydroxyapatite)

- TCP Tricalcium Phosphate 
R\&D AND INNOVATIONS PROPOSED

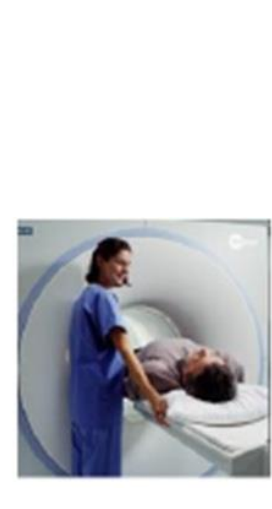

scanning

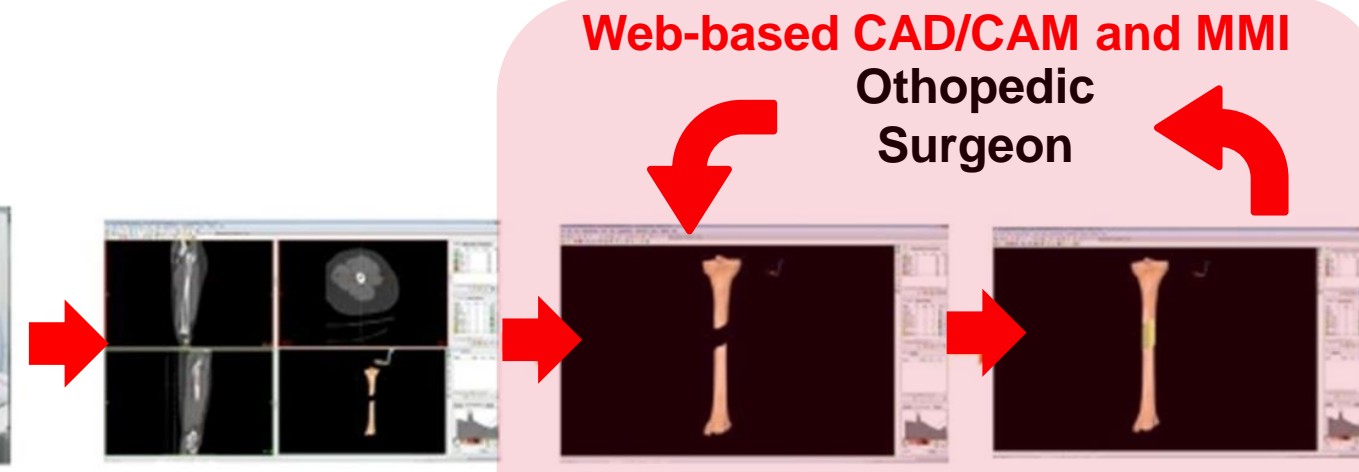

Data processing and CAD generation

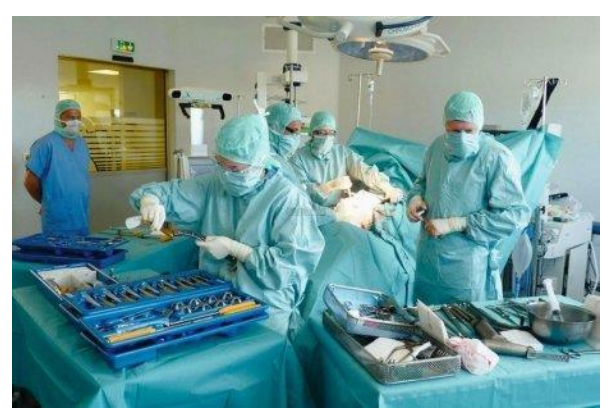

Surgery

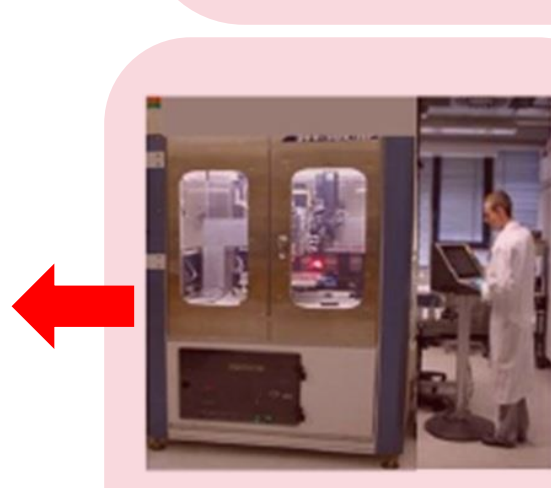

Growing factors, bone cells

Bio-active implant

+ Production of very pure TCP powder of SLM (very low content of metallic elements)

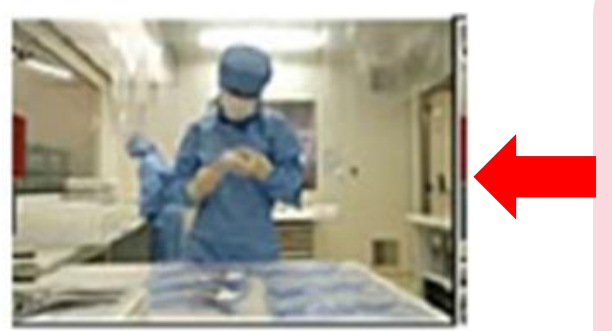

Sterilisation

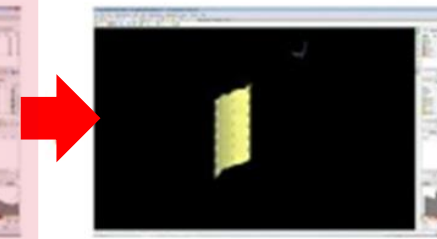

\section{?}



Direct Manufacturing

SLM of HAP or TCP 


\section{TCP POWDER MANUFACTURING}

\section{MANUFACTURING PROCESS OF TRICALCIUM PHOSPHATE B (B-TCP)}

- Chemical synthesis of Apatitic Tricalcium Phosphate

- Conversion into TCP

- Synthesis of macro-powder by spraying

SYNTHESIS OF APATITIC TRICALCIUM PHOSPHATE (TCP AP) Aqueous precipitation

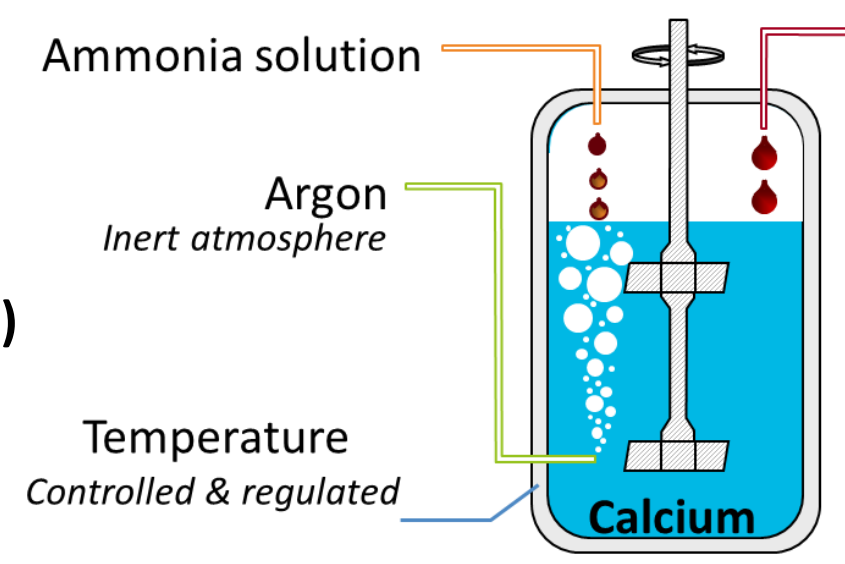

Phosphate

Diammonium hydrogen phosphate $\left(\mathrm{NH}_{4}\right)_{2} \mathrm{HPO}_{4}$

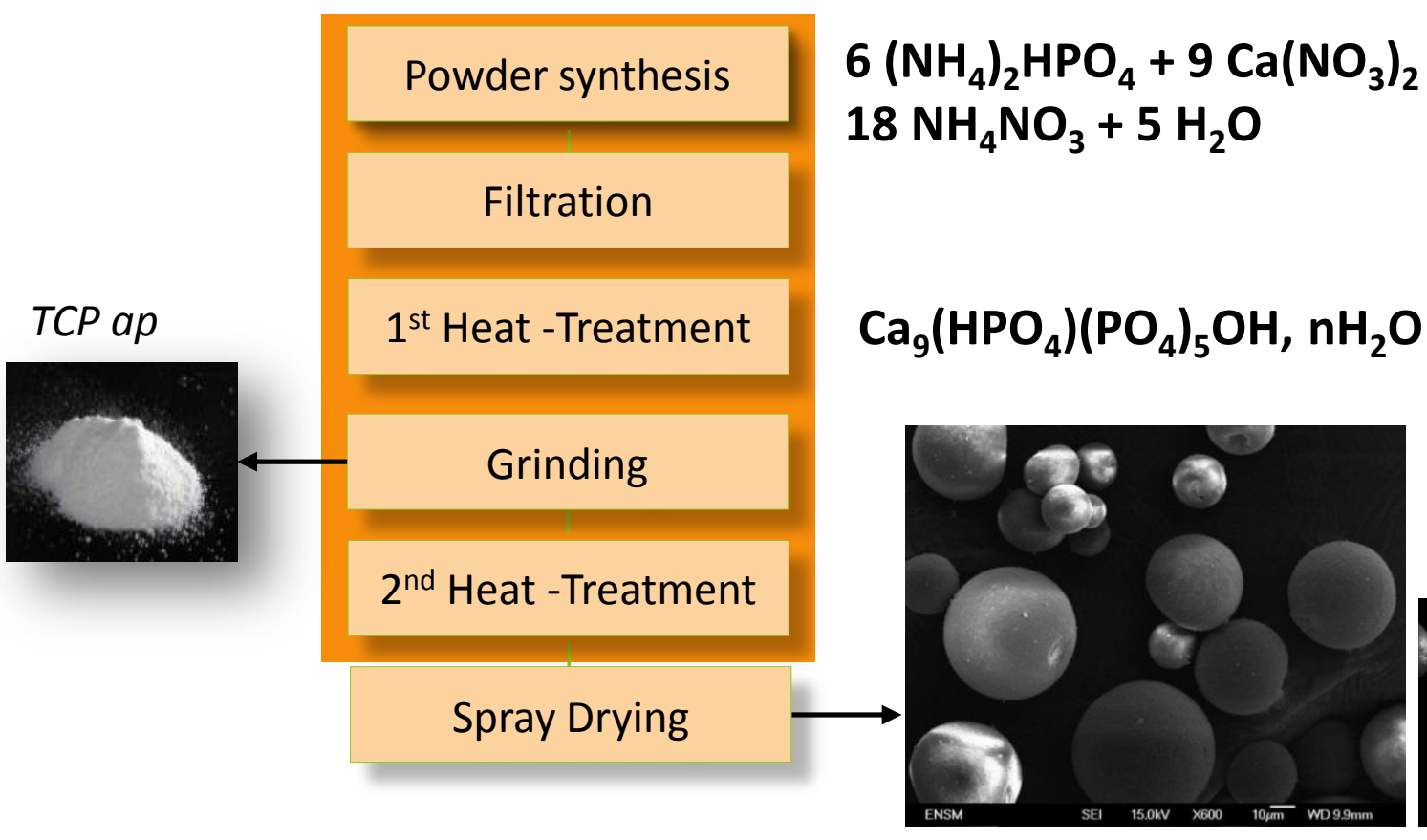
$6\left(\mathrm{NH}_{4}\right)_{2} \mathrm{HPO}_{4}+9 \mathrm{Ca}\left(\mathrm{NO}_{3}\right)_{2}+6 \mathrm{NH}_{4} \mathrm{OH} \rightarrow \mathrm{Ca}_{9}\left(\mathrm{PO}_{4}\right)_{5}\left(\mathrm{HPO}_{4}\right)(\mathrm{OH})+$ Calcium Nitrate Tetrahydrate $\mathrm{Ca}\left(\mathrm{NO}_{3}\right)_{2}$

Powder synthesis

Filtration

$1^{\text {st }}$ Heat - Treatment

Grinding

$2^{\text {nd }}$ Heat -Treatment

Spray Drying

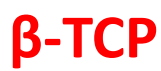

- Ultra pure composition (TCP >99.5\% wt., Metals <50ppm)

- Dense and spherical agregates

- Size distribution: $1 \leq \varnothing \leq 50 \mu \mathrm{m}$



\section{SLM PROCESS PARAMETER SEARCH}

\section{Demonstrator Setup}

\section{SLM demonstrator system}

- Laser : CW (100W), $\mathrm{CO}_{2}$ (50W) (nanoseconde fiber laser, CW Nd:YAG, NIR diode : available)

- Manufacturing Chamber : $\varnothing 100 \mathrm{~mm}$, stainless steel with HAP
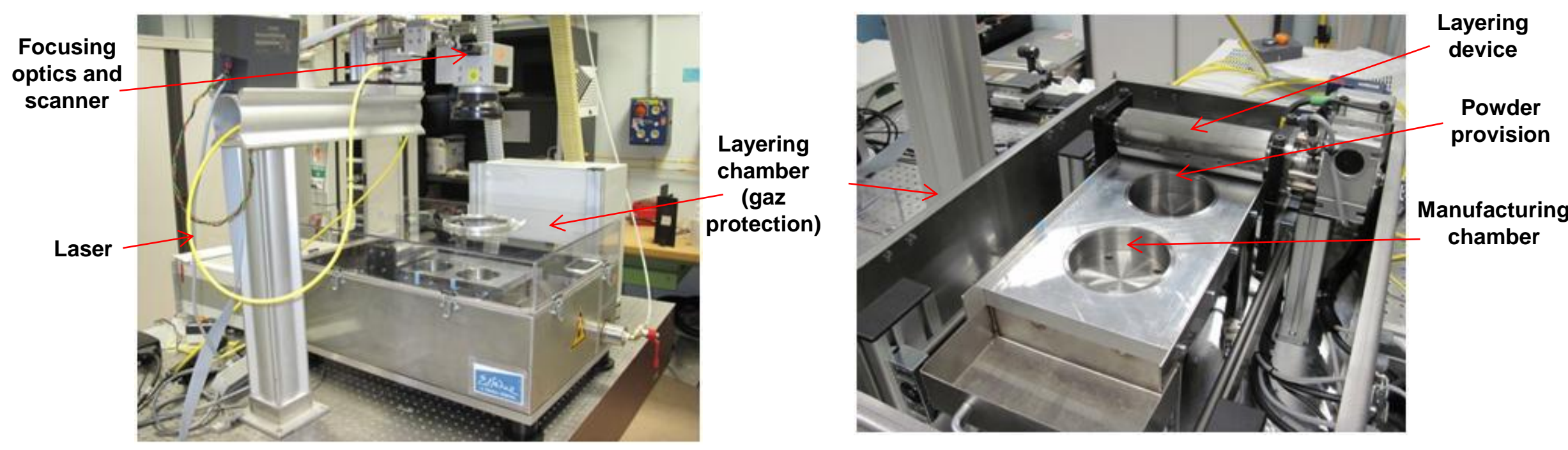

\section{Process parameter search}

- Instrumentation with high speed camera

- Fusion lines

- Main parameters : Power, Scanning speed, Layer thickness, Laser spot diameter,... 


\section{SLM PROCESS STUDY : POWDER ABSORBITIVITY}

\section{ABSORBTIVITY OF TCP POWDER :STATIC TEST}

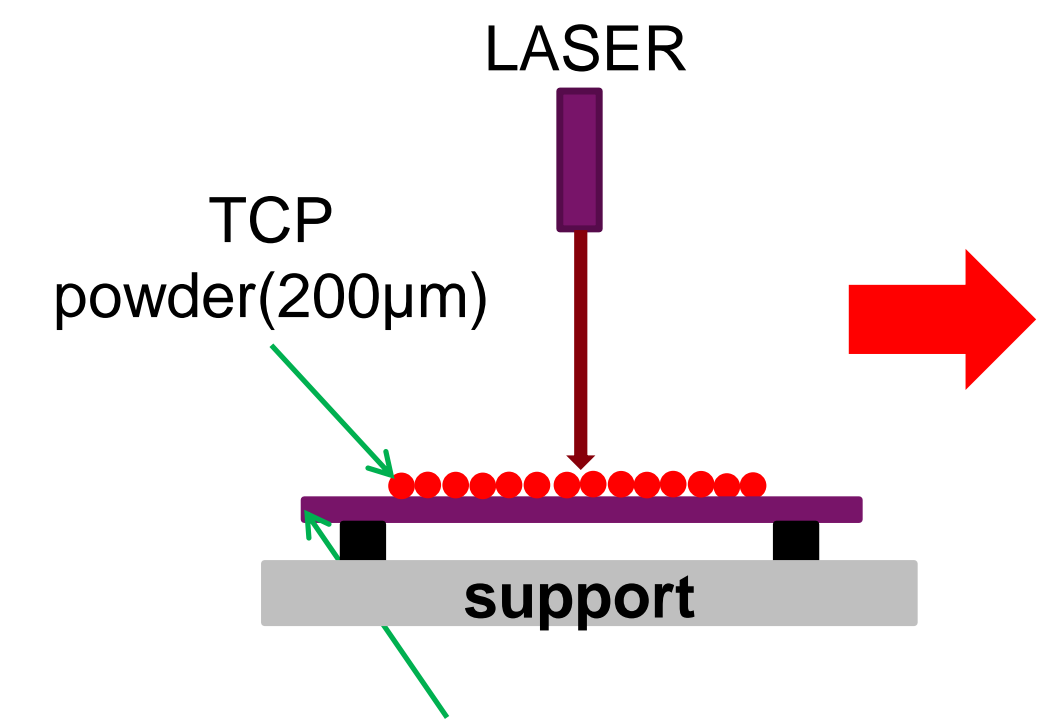

$>838 \mathrm{~nm}$ (Thalès laser diode) (spot $\sim 1 \mathrm{~mm}$ )

$>$ No abosption at $50 \mathrm{~W}$

Transparent material to laser

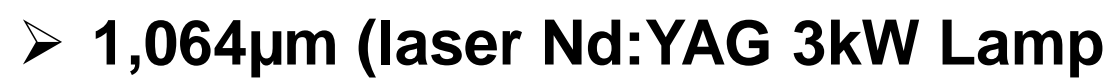

pomped) (spot diam $\sim 1,5 \mathrm{~mm}$ )

$>$ No absorption up to $750 \mathrm{~W}$

$>10,6 \mu \mathrm{m}(\mathrm{CO} 2$ laser 50W max)

$>$ Fusion at few Watts

Study with $\mathrm{CO} 2$ laser possible

For NIR lasers $(1,064 \mu \mathrm{m}, 1.07 \mu \mathrm{m}, 838 \mathrm{~nm}, \ldots)$ : need to modifiy the powder... 


\section{SLM PROCESS STUDY : CW $\mathrm{CO}_{2}$ LASER}

\section{Initial trials}

Powder thickness : $200 \mu \mathrm{m}$

Support : HAP coating

\begin{tabular}{|c|c|c|c|c|c|}
\hline Trial & Power & \multicolumn{2}{|c|}{ Speed } & Foacal spot diam. & T$^{\circ}$ heating \\
\hline TCP_CO2_C1_L4 & $52 \mathrm{~W}$ & $0,1 \mathrm{~m} / \mathrm{s}$ & $6 \mathrm{~m} / \mathrm{min}$ & $200 \mu \mathrm{m}$ & $25^{\circ}$ \\
\hline TCP_CO2_C1_L5 & $52 \mathrm{~W}$ & $0,25 \mathrm{~m} / \mathrm{s}$ & $15 \mathrm{~m} / \mathrm{min}$ & $200 \mu \mathrm{m}$ & $25^{\circ}$ \\
\hline TCP_CO2_C1_L6 & $52 \mathrm{~W}$ & $0,25 \mathrm{~m} / \mathrm{s}$ & $15 \mathrm{~m} / \mathrm{min}$ & $200 \mu \mathrm{m}$ & $25^{\circ}$ \\
\hline TCP_CO2_C1_L7 & $52 \mathrm{~W}$ & $0,05 \mathrm{~m} / \mathrm{s}$ & $3 \mathrm{~m} / \mathrm{min}$ & $200 \mu \mathrm{m}$ & $25^{\circ}$ \\
\hline
\end{tabular}

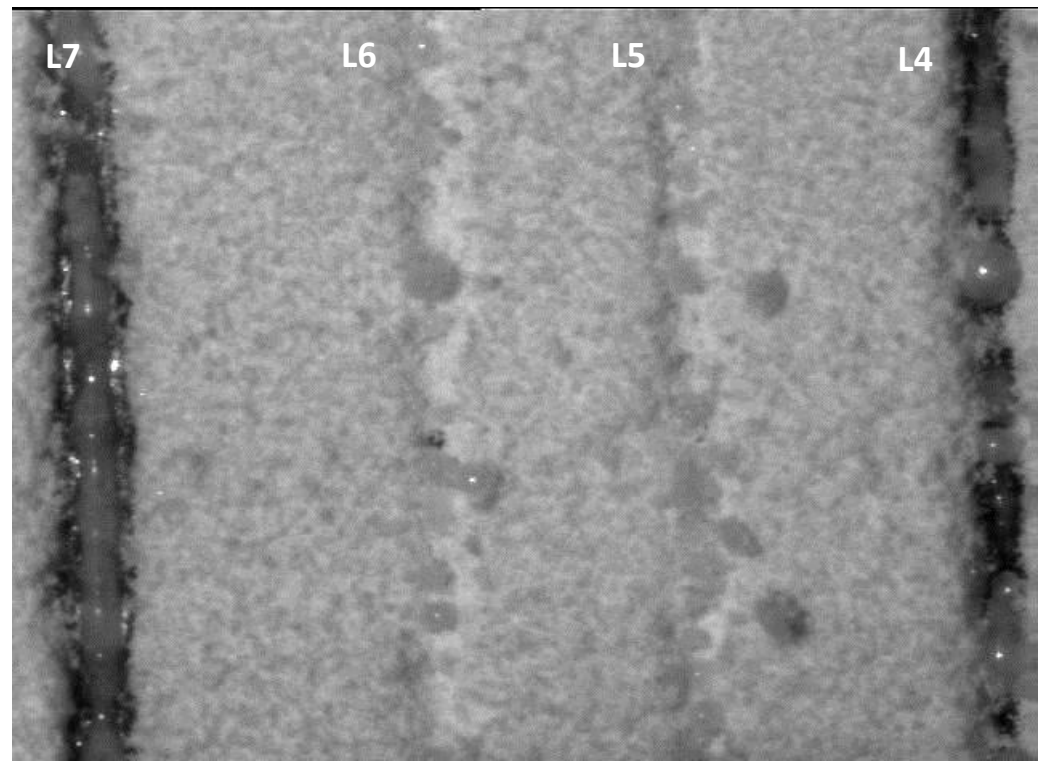
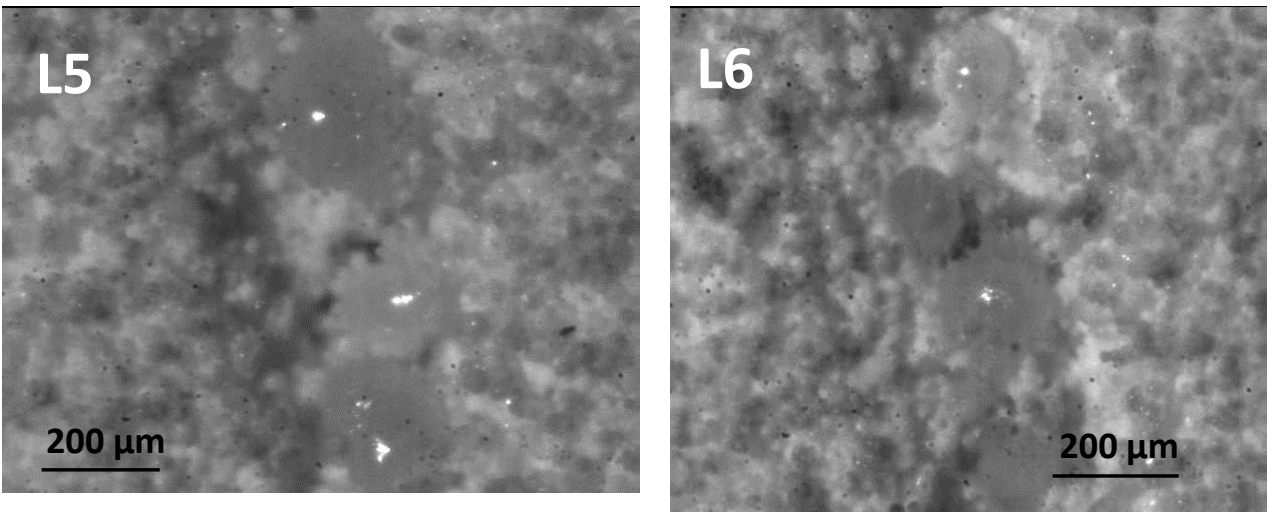

Results :

- Fusion in dropplets for highest speed
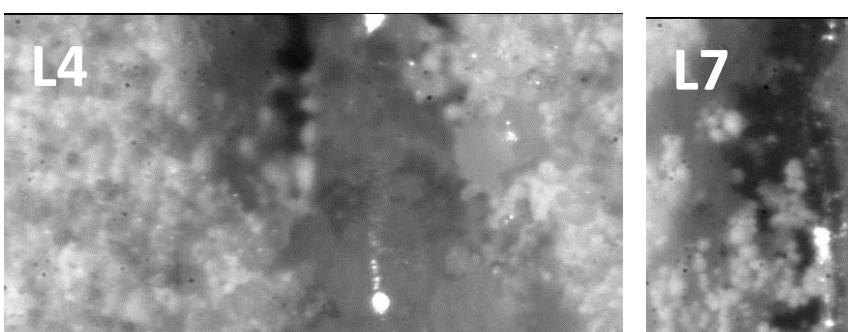

- No enough energy density to get stable fusionTCP

- Need to decrease speed 


\section{SLM PROCESS STUDY : CW $\mathrm{CO}_{2}$ LASER}

\section{Reducing travel speed}

\begin{tabular}{|c|c|c|c|c|}
\hline Trial & Power & Speed & Focal spot diam. & Width \\
\hline TCP_CO2_C1_L1 & $52 \mathrm{~W}$ & $1 \mathrm{~m} / \mathrm{min}$ & $200 \mu \mathrm{m}$ & $\sim 300 \mu \mathrm{m}$ \\
\hline TCP_CO2_C1_L2 & $52 \mathrm{~W}$ & $1.5 \mathrm{~m} / \mathrm{min}$ & $200 \mu \mathrm{m}$ & $\sim 250 \mu \mathrm{m}$ \\
\hline TCP_CO2_C1_L3 & $52 \mathrm{~W}$ & $2 \mathrm{~m} / \mathrm{min}$ & $200 \mu \mathrm{m}$ & $\sim 200 \mu \mathrm{m}$ \\
\hline
\end{tabular}

Powder thickness : $200 \mu \mathrm{m}$

Support : HAP coating

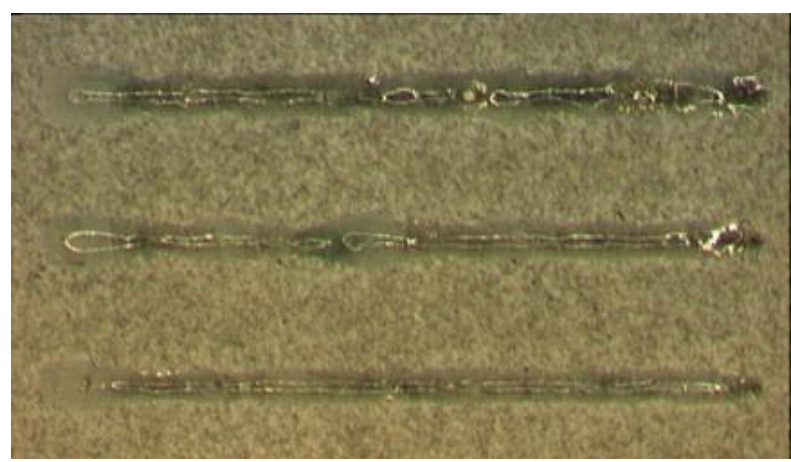

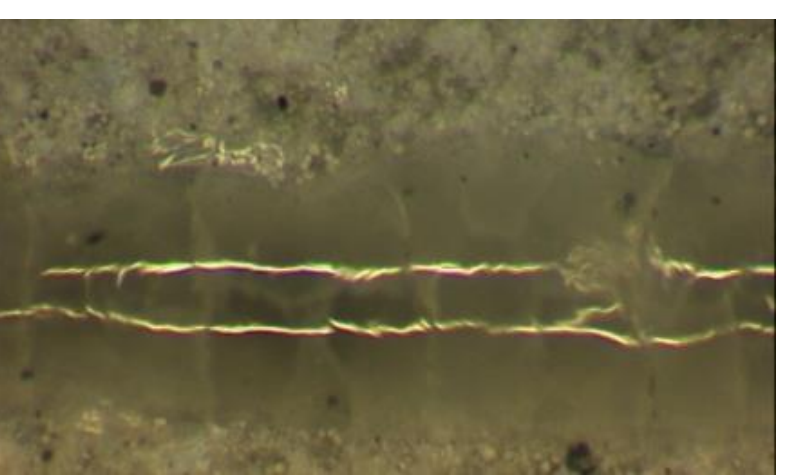

L1 param

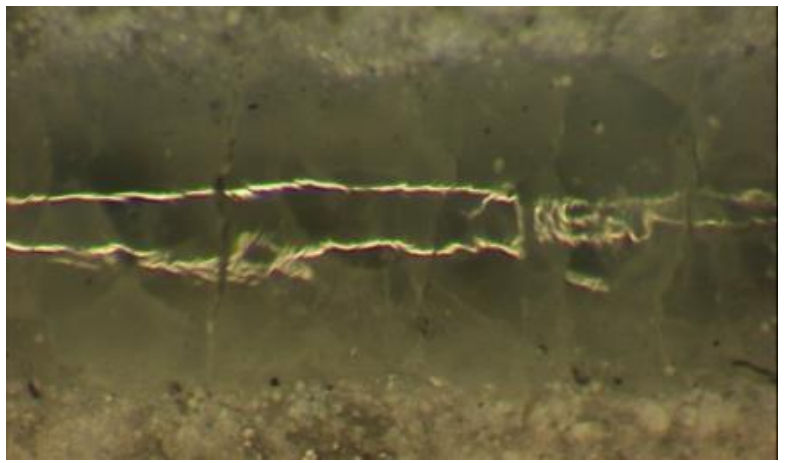

L2 param overview

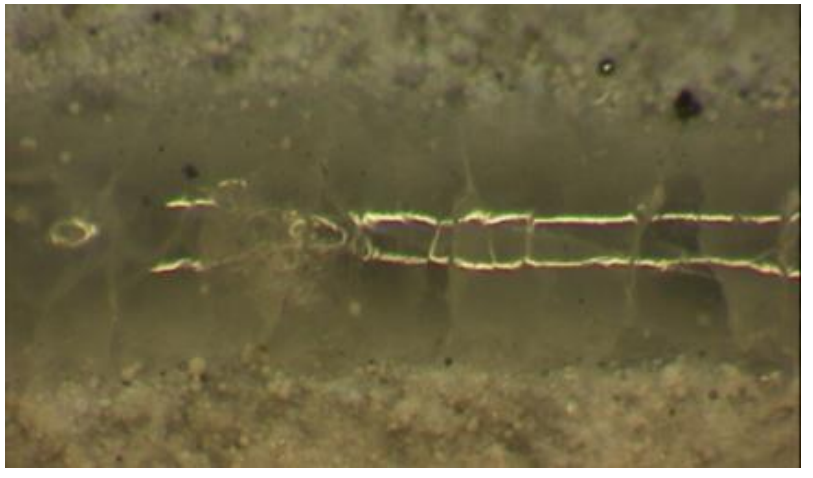

L1 param

\section{Results}

- Generation of continuous and stable fusion of TCP

- Numerous cracks

- Further process parameter optimization

CHeating of manufacturing chamber Need to decrease powder thickness, increase travel speed andlaser power... 


\section{Cea SLM PROCESS STUDY : CW FIBER LASER}

\section{ADDITION OF PARTICLES TO TCP POWDER}

- Biocompatibility

- Sub-micro,nano

$\mathrm{TiC}, \mathrm{SiO}_{2}, \mathrm{SiC} \ldots$

\section{B-TCP+nano SiC}

- First method

Use of $\beta$ TCP powder

Mechanical mixing with nano-SiC powder

1- Second method

Co-spray drying SiC - $\beta$ TCP

- Elaboration of dense and spherical agregates

- Particle size distribution: $\mathbf{1} \leq \varnothing \leq \mathbf{5 0} \boldsymbol{\mu m}$
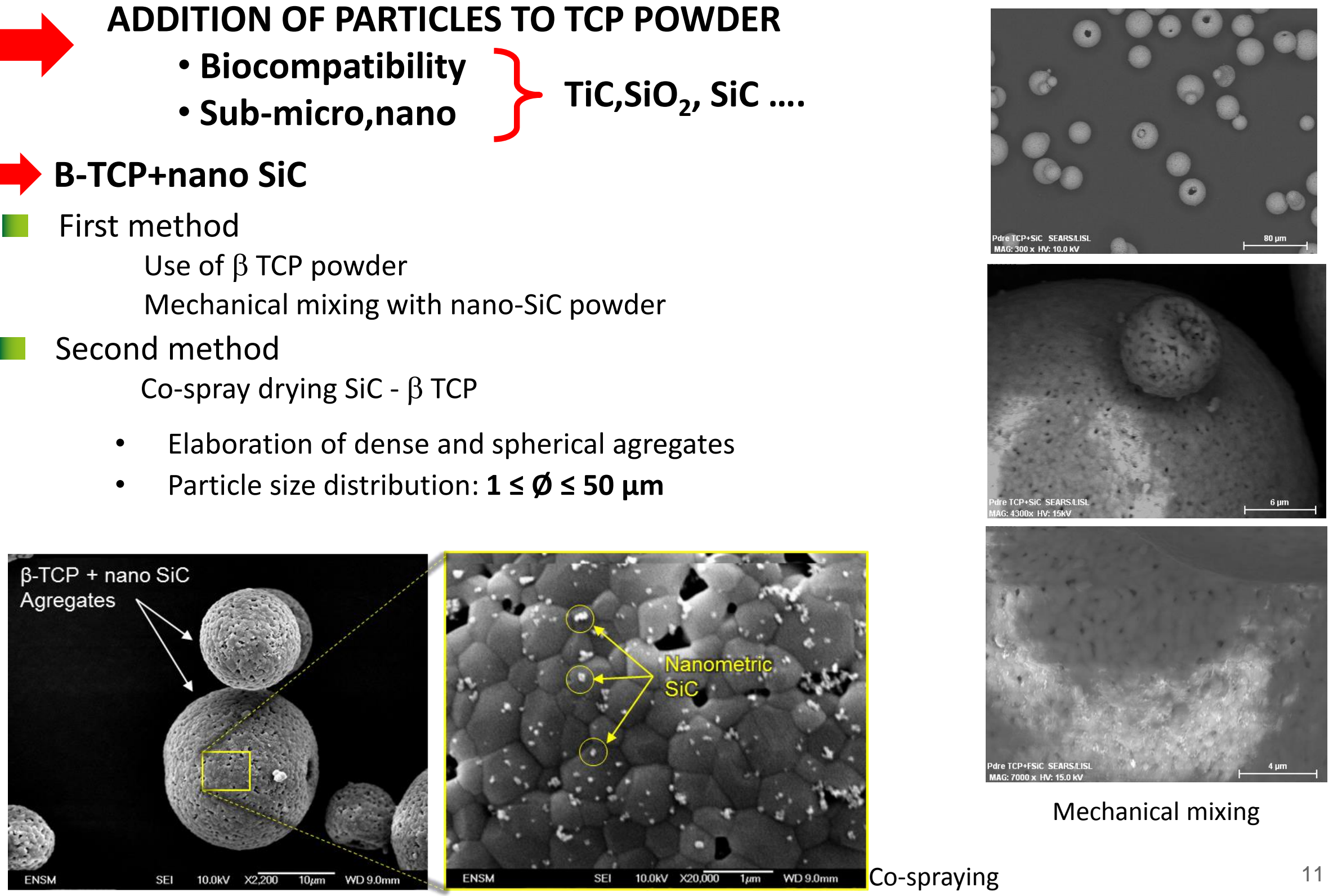

Mechanical mixing 


\section{SLM PROCESS STUDY : CW FIBER LASER}

1. PARAMETER SEARCH FOR 2D LINE ON SUBSTRATE

- HAP coating stainless steel plates

- Power : 30-100W

- Focal spot diameter: $100 \mu \mathrm{m}$

- Scanning speed : $1-200 \mathrm{~mm} / \mathrm{s}$
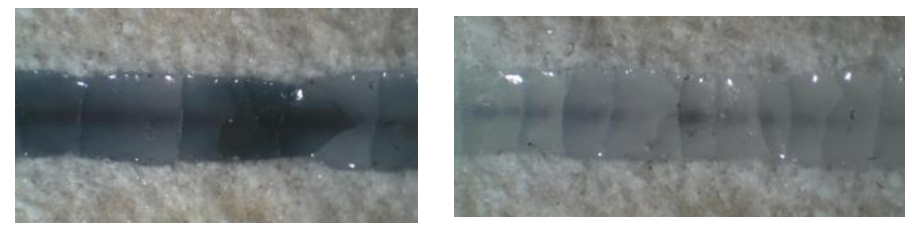

- Selection of best fusion profile (stability)

2. PARAMETER SEARCH FOR 2D SURFACE

- Power : 30-100W

- Focal spot diameter: $1100 \mu \mathrm{m}$

- Scanning speed : $100-200 \mathrm{~mm} / \mathrm{s}$

- Distance between 2D line : $50 \%-100 \%$ of line width and grids

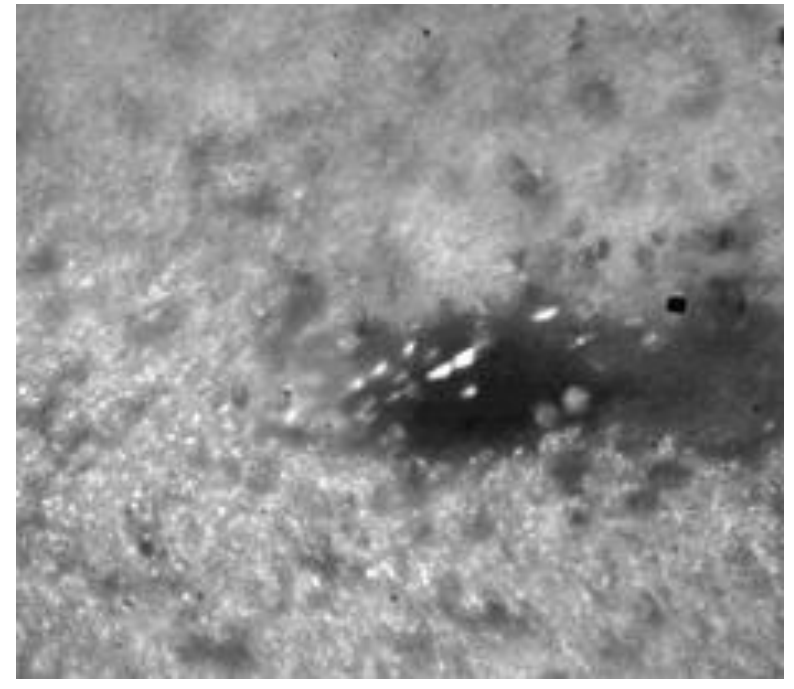

TCP+SiC (f=10kHZ, $P=50 \mathrm{~W}$, $\mathrm{V}=100 \mathrm{~mm} / \mathrm{s}$ )

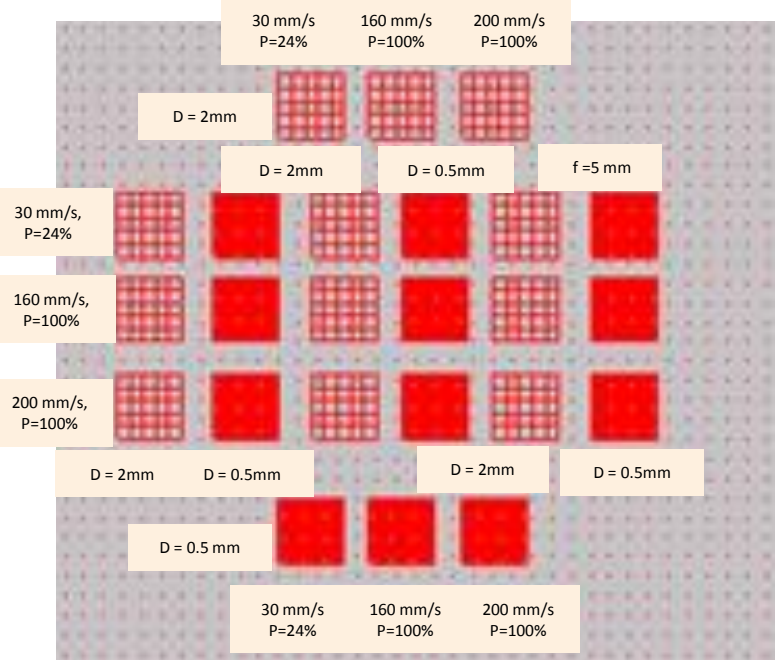




\section{Cea SLM PROCESS STUDY : CW FIBER LASER}

\section{BUILDING 3D SIMPLE GEOMETRIES}

- Squares, cylinders,...

- Focal spot diameter: $100 \mu \mathrm{m}$

- Scanning speed : $1-200 \mathrm{~mm} / \mathrm{s}$

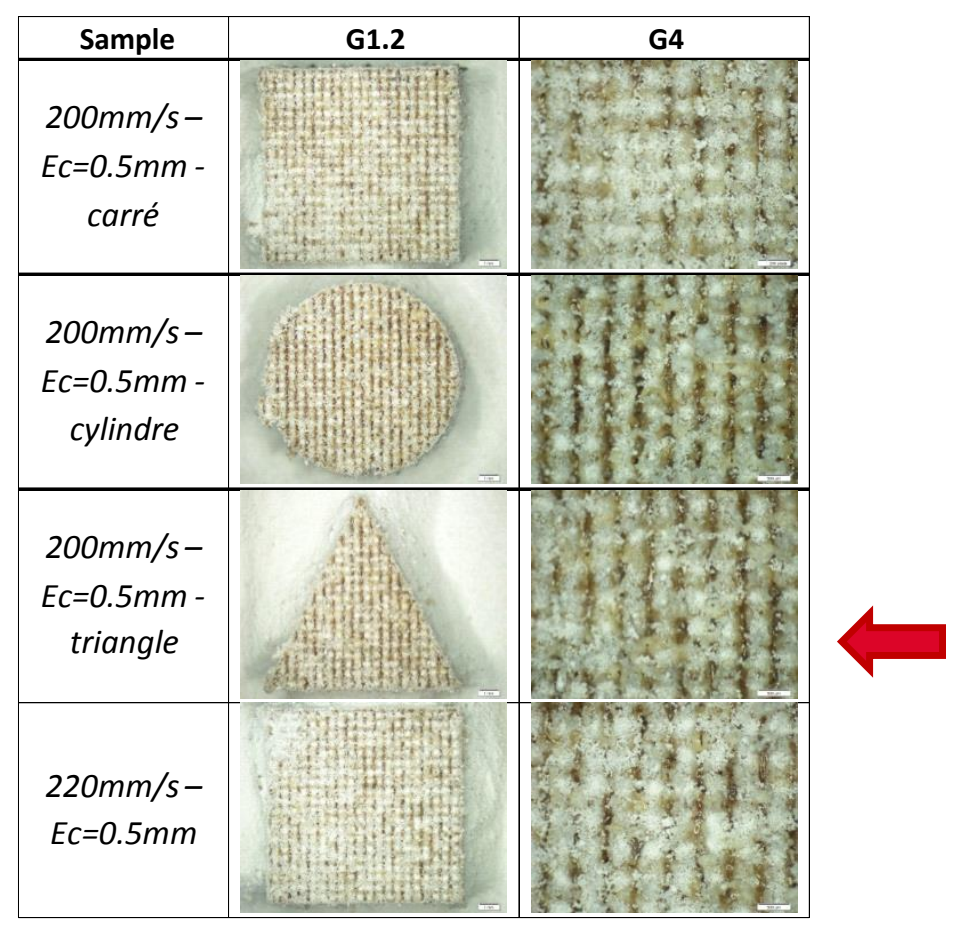
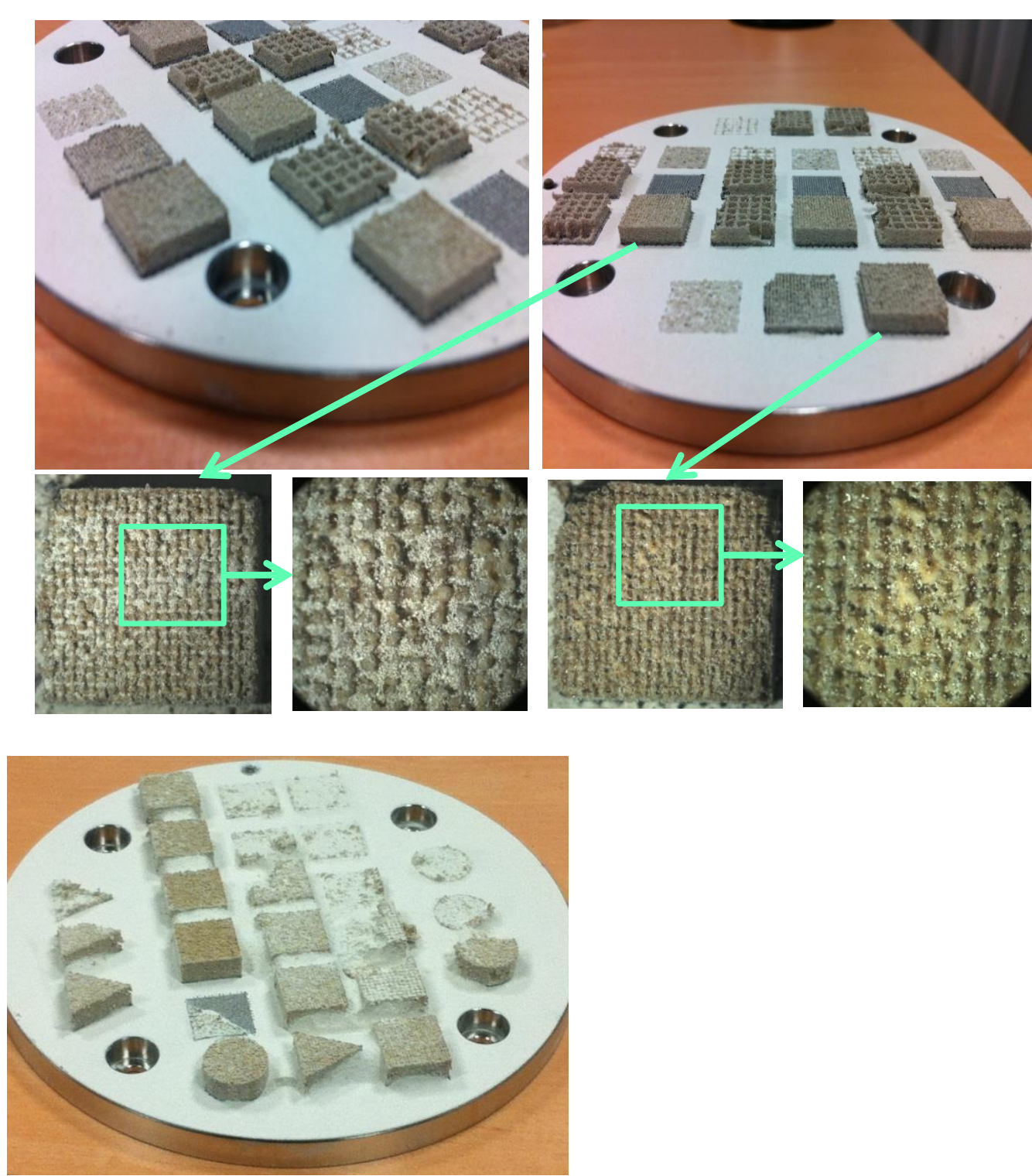


\section{Cea SLM PROCESS STUDY : CW FIBER LASER}

\section{MATERIAL ANALYSIS}
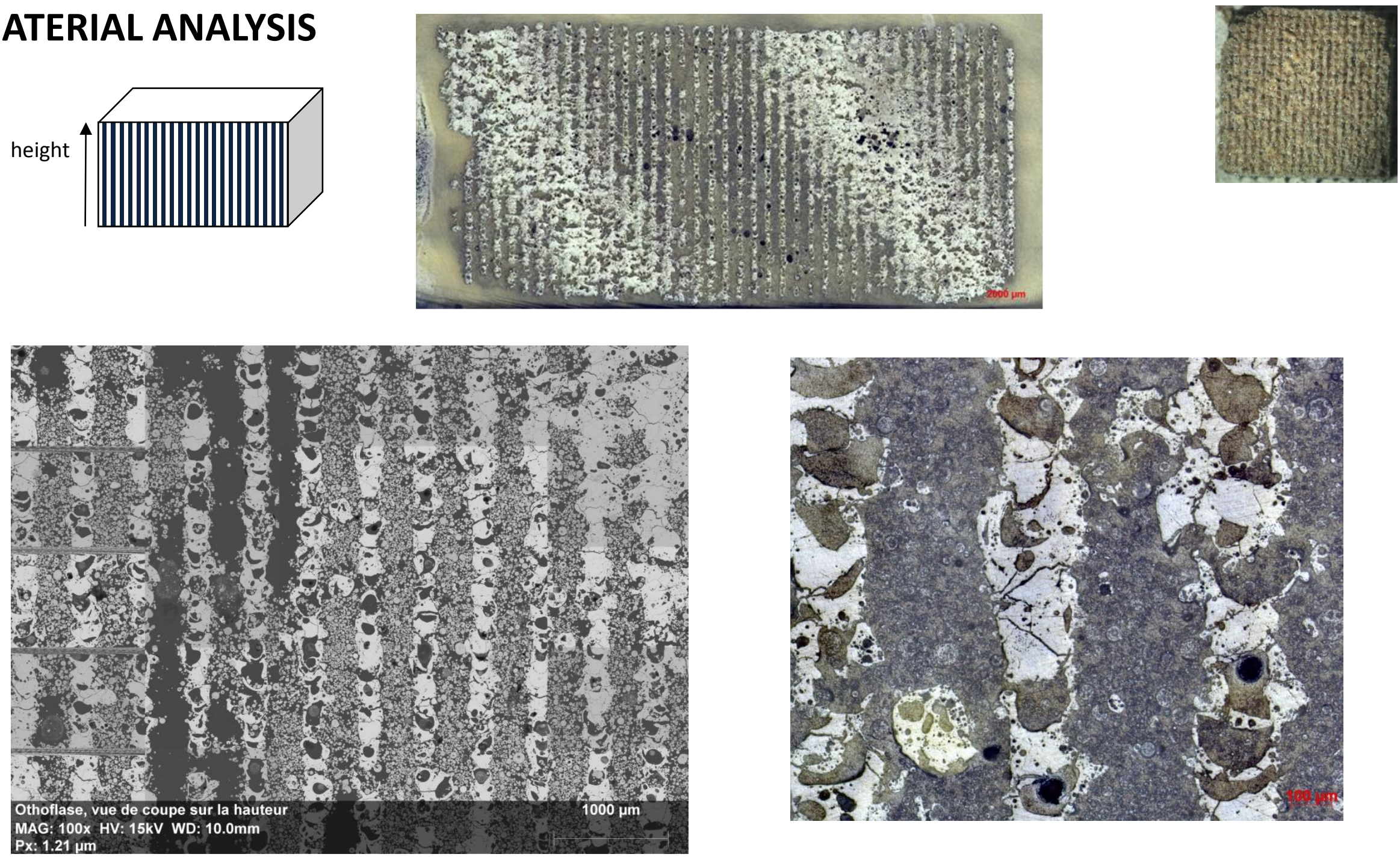


\section{MATERIAL ANALYSIS}
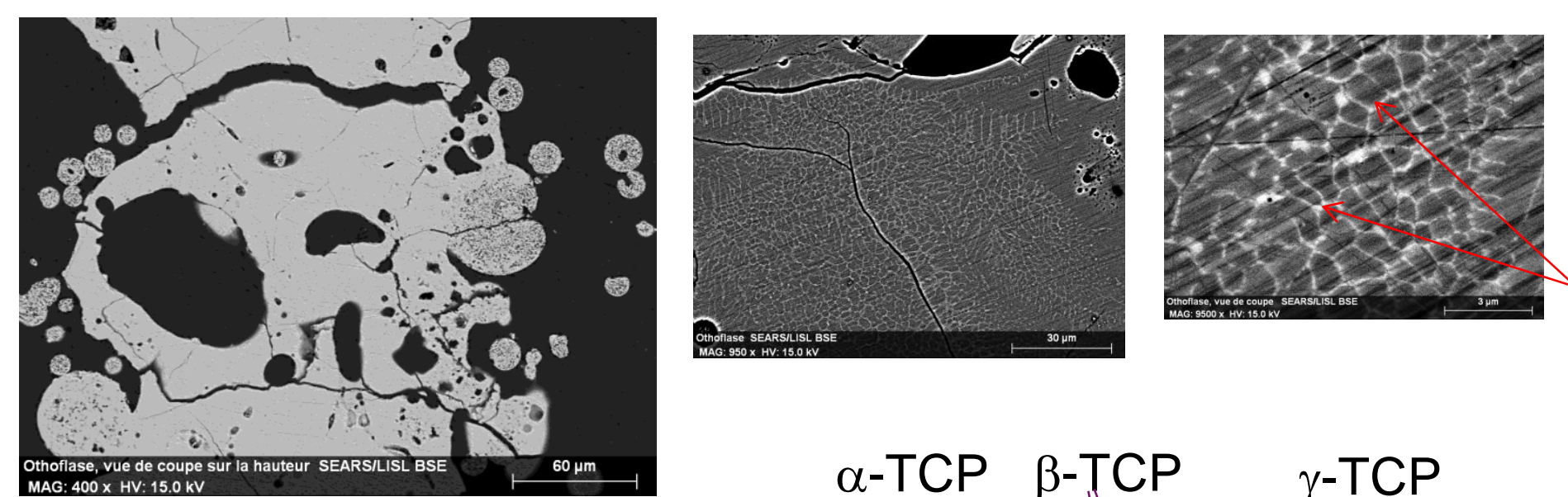

Detail of the microstructure

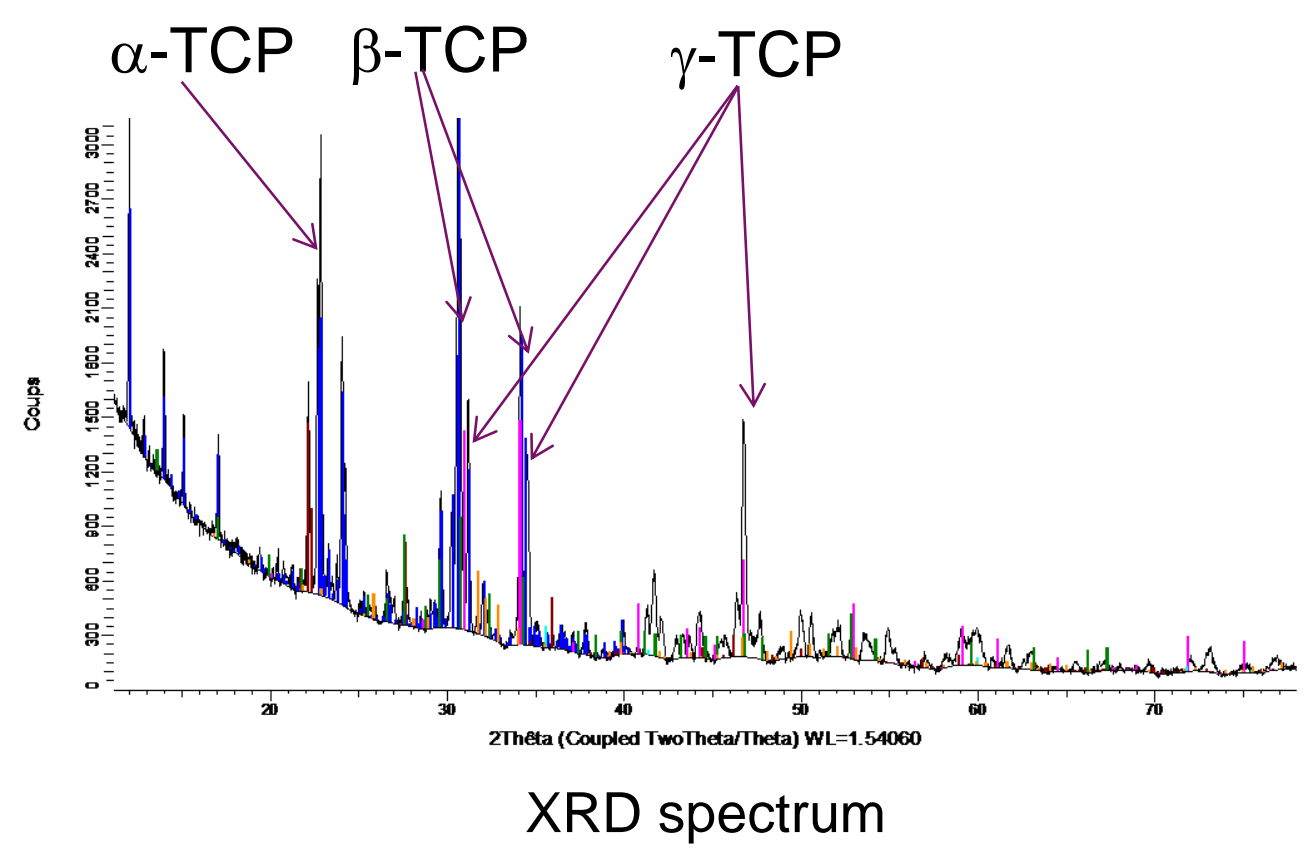

Pure $\beta$-TCP XRD spectrum 


\section{CONCLUSION AND PERSPECTIVES}

\section{Process parameter search}

1. CO2 laser : Continuation of process parameter search for 3D part manufacturing

2. Fiber laser

3. Material study

4. Manufacturing complex part

\section{Biological tests}

1. 3D samples

2. Study of porosity of the sample
- Decrease powder thickness

- Increase of travel speed Decrease of cracks

- Manufacturing of 3D parts

- New additional material

- Optimization of mixture (TCP+add)

Composition Fraction of B-TCP, HAP, TTCP,... 